Original Article

\title{
AMELIORATIVE EFFECT OF HESPERIDIN ON CARBON TETRACHLORIDE INDUCED LIVER FIBROSIS IN RATS
}

\author{
ASMAA RAMADAN ABDEL-STTAR ${ }^{1}$, MARWA MAHMOUD KHALAF², AMIRA M. ABOYOUSSEF², \\ ALI AHMED ABOSAIF ${ }^{1}$
}

1,2Department of Pharmacology and Toxicology, Faculty of Pharmacy, Nahda University, Beni-Suef, Egypt

Email: marwa_mak@yahoo.com

Received: 09 Feb 2017 Revised and Accepted: 19 May 2017

\section{ABSTRACT}

Objective: Exposure to carbon tetrachloride leads to serious liver injury and fibrosis. This study was aimed to evaluate the hepatoprotective effects of hesperidin against carbon tetrachloride $\left(\mathrm{CCl}_{4}\right)$-induced liver fibrosis in rats compared with the reference drug silymarin.

Methods: Wistar albino rats were divided into five groups, each of eight rats. Animals were allocated into a control group, corn oil group and fibrosis control group. The remaining two groups received in addition to $\mathrm{CCl}_{4}$, silymarin $(100 \mathrm{mg} / \mathrm{kg} / \mathrm{d})$ as a reference treatment and hesperidin $(200 \mathrm{mg} / \mathrm{kg} / \mathrm{d})$. At the end of experimental period, the biomarkers of specific fibrosis [hepatic transforming growth factor $\beta 1$ (TGF- $\beta 1$ ) and hydroxyproline (HYP)], liver function [serum alanine transaminase (ALT), aspartate transaminase (AST), albumin and total bilirubin], oxidative stress [hepatic malondialdehyde (MDA), glutathione (GSH) and catalase (CAT)], inflammatory [hepatic myeloperoxidase (MPO), serum tumor necrosis factor alpha (TNF- $\alpha$ )], relative liver weight, lipid profile [total cholesterol, serum triglycerides, high-density lipoprotein cholesterol (HDL$\mathrm{Ch}$ ) and low density lipoprotein cholesterol (LDL-Ch)] were evaluated, supported by liver histopathological study and immunohistochemistry of alpha-smooth muscle actin ( $\alpha$-SMA) in liver sections.

Results: Hesperidin significantly decreased hepatic transforming growth factor $\beta_{1}$, hydroxyproline, the serum liver function markers of ALT, AST and total bilirubin, the hepatic content of MDA and MPO activity, the serum pro-inflammatory cytokine TNF- $\alpha$, relative liver weight, and the serum lipid profile markers cholesterol, triglycerides and LDL. On the other hand, Hesperidin significantly increased albumin, the hepatic content of GSH and CAT, and serum lipid profile of LDL. In addition, liver sections obtained from these groups showed marked histopathological and immunohistochemistry of $\alpha$-SMA improvement.

Conclusion: Hesperidin may be promising protective agent against liver fibrosis through improvement of liver function, modulation of the fibrous scar formation, anti-inflammatory and antioxidant potentials.

Keywords: Liver fibrosis, $\mathrm{CCl}_{4}$, Corn oil, Silymarin, Hesperidin, Rat

(c) 2017 The Authors. Published by Innovare Academic Sciences Pvt Ltd. This is an open access article under the CC BY license (http://creativecommons.org/licenses/by/4.0/) DOI: http://dx.doi.org/10.22159/ijpps.2017v9i7.17611

\section{INTRODUCTION}

Hepatic fibrosis (HF) is a great health problem unless controlled it can progress to cirrhosis and primary liver cancer, which are responsible for most liver transplants and deaths [1]. HF is a healing respond of liver tissue damage. It is a reversible process and marked by over accumulation of extracellular matrix (ECM) protein [2]. The imbalance between enhanced ECM synthesis and decreased breakdown of connective tissue proteins will damage the normal architecture of the liver, which changes the normal function of the organ [3].

When the hepatic stellate cell (HSC) is activated, it loses its retinoid and starts expressing new receptors such as the platelet-derived growth factor (PDGF) receptor and transforming growth factor (TGF $\beta_{1}$ ) receptor. It also expresses new proteins such as $\alpha$-smooth muscle actin. The activated HSC proliferates and synthesises extracellular matrix proteins to produce the fibrous scar [4].

Free radical-initiated lipid peroxidation plays a role in hepatic fibrogenesis, which affected the cellular permeability of hepatocytes leading to increasing levels of liver enzymes [5]. Therefore, there is a possible role of antioxidants in the prevention and treatment of liver diseases [6]. The level of liver hydroxyproline (HYP) reflects the amount of hepatic collagen which makes it an important marker of liver fibrosis to be determined in liver tissue. It has been previously reported that $\mathrm{CCl}_{4}$ intoxication leads to elevation of lipid biomarkers and accumulation of fat vacuoles, which may reflect impairment of liver function, particularly on lipid metabolism [7].

Until now, there is no standard treatment for liver fibrosis. In addition, the current therapies are often ineffective in treating the attached causes of fibrosis and are associated with many side effects. Therefore, there is a great demand for new drug classes to be proved as potent and safe antifibrotic agents, aiming at least to prevent the progression to end-stage liver disease [6].

Hesperidin (3,5,7-trihydroxy flavanone-7-rhamnoglucoside) [8], a flavanone glycoside present abundantly in citrus fruits [9]. This flavonoid has potential therapeutic benefits including, antiviral, antiallergic, antiplatelet, anti-inflammatory and antioxidant activities [10].

Silymarin is an extract from milk thistle Silybum marianun. It is used as hepatoprotective drug based on its free radical scavenging, antiinflammatory and antifibrotic activities. Silymarin has clinical applications in liver fibrosis, liver cirrhosis and drug-induced liver diseases [11].

Based on this background, the present study aimed to evaluate the possible hepatoprotective effects of hesperidin, as compared to the reference drug silymarin, on experimentally-induced HF in adult male Wistar albino rats.

\section{MATERIALS AND METHODS}

Materials

Animals

This study was performed on healthy adult male Wistar albino rats, weighing $250 \pm 10 \mathrm{~g}$. Animals were obtained from Animal House of Faculty of Pharmacy, Nahda University, Beni-Suef, Egypt and they were housed in the air-conditioned pathogen-controlled animal room in the animal house. All experimental rats were kept under stable temperature $\left(25 \pm 1{ }^{\circ} \mathrm{C}\right)$ and relative humidity and allowed free access to standard 
forage and tap water ad libitum. All procedures performed in studies involving animals were in accordance with the ethical standards of the National Institutes of Health (NIH) Guide for Care and Use of Laboratory Animals (Publication No. 85-23, revised 1985).

\section{Drugs, chemicals and reagent kits}

$\mathrm{CCl}_{4}$ and corn oil were purchased from El-Nasr Chemical Company (Abou-Zaabal, Cairo, Egypt). Silymarin was purchased from SigmaAldrich Chemical Company (St. Louis, USA). Hesperidin was purchased from Acros Organics Company (New Jersery, USA). Tissue enzymelinked immune sorbent assay (ELISA) kits of transforming growth factor $\beta 1$ (TGF- $\beta 1$ ) was obtained from MyBioSource, Inc. (San Diego, USA). Serum ELISA tumour necrosis factor alpha (TNF- $\alpha$ ) kit was supplied by RandD Systems (McKinley, Minneapolis, United States). Tissue colorimetric kit of myeloperoxidase (MPO) was purchased from Amsbio Company (Milton Park, Abingdon, UK). Tissue colorimetric antioxidant kits of malondialdehyde (MDA), glutathione (GSH) and catalase (CAT) were purchased from Bio-Diagnostic Company (Dokki, Giza, Egypt). Serum colorimetric kits of liver function serum alanine transaminase (ALT), aspartate transaminase (AST), albumin, total bilirubin and lipid profile [total cholesterol, triglycerides (TGs) and high-density lipoprotein cholesterol (HDL-Ch)] were purchased from BioMed company (Badr City, Industrial Area Piece, Egypt). All others chemicals, solvents and reagents used were of the highest analytical grade commercially available.

\section{Experimental design}

Rats were randomly allocated into 5 weight-matched groups, each of 8 rats. The first group was kept as a normal control group and received only saline $5 \mathrm{ml} / \mathrm{kg} / \mathrm{d}$, p. o. The second group was kept as a corn oil group and received only corn oil $1 \mathrm{ml} / \mathrm{kg}$, i. p. two times per week for five weeks. The remaining three groups received intraperitoneal $\mathrm{CCl}_{4}$ in corn oil (1:1) at a dose of $2 \mathrm{ml} / \mathrm{kg}$, i. p. [12] one of them was left as fibrosis control group. The remaining 2 groups received the following treatments; silymarin $100 \mathrm{mg} / \mathrm{kg} / \mathrm{d}$, p. o. [13] as a reference treatment and hesperidin $200 \mathrm{mg} / \mathrm{kg} / \mathrm{d}$, p. o. [9], respectively. All the treatments were given daily for five consecutive weeks starting from the first day of induction. Doses of test agents were determined with pilot trials guided by the published literature. Blood and liver tissue samples were withdrawn $24 \mathrm{~h}$ after the last dose.

\section{Methods}

\section{Induction of liver fibrosis}

Induction of liver fibrosis was performed by i. p. injection of rats with $\mathrm{CCl}_{4}$ in corn oil $(1: 1)$ at a dose of $2 \mathrm{ml} / \mathrm{kg}$, twice weekly for five weeks according to Hui et al. [12].

\section{Serum preparation}

At the end of the experiment, rats were anaesthetized with light ether. Blood samples were withdrawn from the retro-orbital plexus in centrifuge tubes using heparinized micro capillary tubes. After collecting blood samples, the tubes were allowed to coagulate at room temperature. Then samples were centrifuged for $20 \mathrm{~min}$ at $4000 \mathrm{rpm}$ using a cooling centrifuge (Sigma 3-30 k, USA). The clear serum layer was separated and stored at $-80^{\circ} \mathrm{C}$ in a deep freezer (Als Angelantoni Life Science, Italy) for the analysis of ALT, AST, albumin, bilirubin, cholesterol, triglycerides, HDL, LDL and TNF- $\alpha$.

\section{Calculation of relative liver weight}

Firstly, the animals have been weighed just before killing. Then, they were rapidly killed by cervical dislocation soon after blood samples were collected. Abdominal cavities were opened, the whole liver tissues were carefully isolated and washed several times with $0.9 \%$ sterile ice-cooled saline to remove any blood from the tissues and then pressed between 2 filter papers to absorb the excess saline solution. Each liver was weighed.

\section{CALCULATION}

$$
\text { Relative liver body weight }=\frac{\text { Weight of liver }(\mathrm{gm})}{\text { Body weight of rat }(\mathrm{gm})} \times 100
$$

\section{Preparation of tissue homogenate}

Livers were cut into small portions and used for the preparation of liver homogenates and histopathology sections. A portion of the liver was homogenized with 5 volumes of isotonic ice-cold normal saline using a homogenizer (Ultra-Turrax $\mathrm{T} 25$, made in Germany), to prepare $20 \%$ liver homogenate. Aliquots of liver homogenates (20\%) were centrifuged at $4000 \mathrm{rpm}$ for $15 \mathrm{~min}$ at $4{ }^{\circ} \mathrm{C}$ and the supernatant was collected, and then stored at $-80{ }^{\circ} \mathrm{C}$ for analysis of HYP, TGF $\beta_{1}$, MDA, GSH, CAT and MPO.

\section{Assessment of serum biomarkers}

Serum ALT and AST levels were assayed according to the method described by Reitman and Frankel [14]. Serum albumin and bilirubin were assessed as described by Doumas et al., Malloy and Evelyn [15, 16], respectively. Serum TNF- $\alpha$ was assessed as described by Brouckaert et al. [17]. Serum total cholesterol, TGs and HDL were estimated by the method of Watson, Fossati and Prencipe, and Castelli et al. $[18,19,20]$ and respectively, while LDL was assessed according to Friedewald et al. [21].

Formula: $\mathrm{LDL}=\mathrm{TC}-\mathrm{HDL}-(\mathrm{TG} / 5.0) \mathrm{mg} / \mathrm{d} l$.

\section{Assessment of liver homogenate biomarkers}

Hepatic TGF- $\beta 1$ was assessed by ELISA kit based on the principle previously described by Blanchette et al. [22]. Hepatic HYP was assessed as described by Patiyal and Katoch [23]. Hepatic MDA, GSH and CAT levels were measured colorimetrically according to Satoh, Beutler et al. and Aebi $[24,25,26]$, respectively, while MPO was assessed as described by Weiss et al. [27].

\section{Histopathological and immunohistochemical study}

Samples were taken from the isolated livers of rats in different groups and immediately fixed in $10 \%$ formalin solution in normal saline and embedded in paraffin. Paraffin beeswax tissue blocks were prepared for sectioning at 4-5 microns. Sections of samples were collected on glass slides, deparaffinized, stained by hematoxylin and eosin stain for routine examination then examination was done through the light electric microscope.

Alpha-smooth muscle actin ( $\alpha$-SMA) was detected immunohistochemically as a marker of HSC activation. Paraffinembedded tissue sections of $3 \mu \mathrm{m}$ thickness were rehydrated first in xylene and then in graded ethanol solutions. The slides were then blocked with $5 \%$ bovine serum albumin (BSA) in Tris-buffered saline (TBS) for $2 \mathrm{~h}$. The sections were then immunostained with anti $\alpha$-SMA antibody (Santa-Cruz Biotechnology, California, USA) at a concentration of $1 \mu \mathrm{g} / \mathrm{ml}$ containing 5\% BSA in TBS and incubated overnight at $4{ }^{\circ} \mathrm{C}$. After washing the slides with TBS, the sections were incubated with goat anti-rabbit secondary antibody. Sections were then washed with TBS and incubated for 5-10 min in a solution of $0.02 \%$ diaminobenzidine containing 0.01\% H2O2. Counterstaining was performed using hematoxylin, and the slides were visualized under a light microscope. Sections of samples were investigated by the aid of two experienced pathologists blinded to the experiment.

\section{Statistical analysis}

All numerical data were expressed as means of 8 values \pm standard error of the mean (SEM). Statistical analysis was performed using one-way analysis of variance (ANOVA) test followed by TukeyKramer multiple comparisons test by the aid of the statistical package for social sciences (SPSS; version 22.0) computer software program (SPSS Inc., Chicago, IL, USA), where the value of $p<0.05$ was considered statistically significant.

\section{RESULTS}

\section{Biochemical estimations}

Effect of silymarin or hesperidin on serum level of liver function markers

$\mathrm{CCl}_{4}$ significantly increased the serum level of ALT, AST and total bilirubin, while the serum level of albumin was significantly decreased as compared with control and corn oil groups. 
Silymarin or hesperidin significantly reduced the serum level of ALT, AST and total bilirubin and significantly increased serum level of albumin as compared to the fibrosis control group.

Hesperidin treatment restored serum ALT and AST back to normal levels, while silymarin or hesperidin treatments restored serum bilirubin back to normal level (table 1).

\section{Effect of silymarin or hesperidin on hepatic content of oxidative} markers

Fibrosis control group showed a significant increase in the hepatic content of MDA and a significant decrease in hepatic GSH content and CAT activity as compared to normal control and corn oil rats.

Treatment of rats with silymarin or hesperidin significantly reduced the hepatic content of MDA and increased GSH and CAT as compared to the fibrosis control group (table 2).

Effect of silymarin or hesperidin on serum and tissue inflammatory markers

$\mathrm{CCl}_{4}$ treated group showed a significant increase in serum level of TNF- $\alpha$, the hepatic content of the inflammatory marker MPO and the relative liver weight as compared to the control and corn oil groups.

Treatment of rats with silymarin or hesperidin significantly reduced the serum level of TNF- $\alpha$, the hepatic content of MPO and the relative liver weight as compared to the fibrosis control group.
Hesperidin showed significantly better improvements of MPO and TNF- $\alpha$ as compared to the reference silymarin and restored MPO back to normal levels (table 3 ).

Effect of silymarin or hesperidin on serum level lipid profile markers

$\mathrm{CCl}_{4}$ showed a significant increase in serum total cholesterol, TGs and LDL levels, while a significant decrease in serum level of HDL as compared to normal control and corn oil groups.

Treatment of rats with silymarin or hesperidin significantly reduced serum total cholesterol, TGs and LDL levels and significantly increased serum level of HDL as compared to the fibrosis control group.

Hesperidin restored cholesterol back to normal levels, while silymarin or hesperidin restored TGs back to normal levels (table 4).

Effect of silymarin or hesperidin on hepatic content of specific fibrosis markers

Rats subjected to $\mathrm{CCl}_{4}$ treatment showed a significant increase in the hepatic content of TGF- $\beta 1$ and HYP when compared with control and corn oil groups.

Silymarin or hesperidin significantly decreased the hepatic content of TGF- $\beta 1$ and HYP as compared with fibrosis control group.

Silymarin or hesperidin treatments were restored hepatic contents of hydroxyproline back to normal level (fig. 1 and 2).

Table 1: Effect of silymarin or hesperidin on serum levels of ALT, AST, albumin or total bilirubin in rats with experimentally-induced liver fibrosis

\begin{tabular}{lllll}
\hline $\begin{array}{l}\text { Parameters } \Rightarrow \\
\text { Groups } \mathbb{}\end{array}$ & ALT (u/l) & AST (u/l) & Albumin (gm/dl) & Bilirubin (mg/d) \\
\hline Control (5 ml saline/kg/p. o.) & $18.0 \pm 1.2$ & $13.1 \pm 1.1$ & $4.7 \pm 0.4$ & $1.0 \pm 0.1$ \\
Corn oil (1 ml/kg/i. p.) & $14.6 \pm 1.4$ & $15.0 \pm 0.8$ & $5.0 \pm 0.2$ & $0.9 \pm 0.1$ \\
Fibrosis control CCl 4 with corn oil (1:1) & $72.5 \pm 7.3^{\mathrm{a}, \mathrm{b}}$ & $54.3 \pm 2.8^{\mathrm{a}, \mathrm{b}}$ & $1.4 \pm 0.1^{\mathrm{a}, \mathrm{b}}$ & $1.9 \pm 0.2^{\mathrm{a}, \mathrm{b}}$ \\
(2 ml/kg/i. p.) & & & & \\
Silymarin (100 mg/kg/p. o.) & $42.5 \pm 3.6^{\mathrm{a}, \mathrm{b}, \mathrm{c}}$ & $26.8 \pm 2.2^{\mathrm{a}, \mathrm{b}, \mathrm{c}}$ & $2.9 \pm 0.1^{\mathrm{a}, \mathrm{b}, \mathrm{c}}$ & $1.1^{\mathrm{c}} \pm 0.1^{\mathrm{c}}$ \\
Hesperidin (200 mg/kg/p. o.) & $24.5 \pm 1.7_{\mathrm{c}, \mathrm{d}}$ & $20.2 \pm 2.0^{\mathrm{c}}$ & $3.4 \pm 0.3^{\mathrm{a}, \mathrm{b}, \mathrm{c}}$ & $1.0 \pm 0.1^{\mathrm{c}}$ \\
\hline
\end{tabular}

Each value represents the mean of 8 animals \pm standard error of the mean (SEM), aSignificantly different from control group value at $p<0.05$, ${ }^{b}$ Significantly different from corn oil group value at $p<0.05$, cSignificantly different from fibrosis control group value at $p<0.05$ and dSignificantly different from silymarin reference treated group value at $p<0.05$

Table 2: Effect of silymarin or hesperidin on liver contents of MDA, GSH and liver CAT activity in rats with experimentally-induced liver fibrosis

\begin{tabular}{llll}
\hline $\begin{array}{l}\text { Parameters } \Rightarrow \\
\text { Groups } \mathbb{}\end{array}$ & MDA (nmol/mg ptn) & GSH (u/mg ptn) & CAT (mmol/mg ptn) \\
\hline Control (5 ml saline/kg/p. o.) & $1.2 \pm 0.1$ & $63.8 \pm 1.6$ & $117.5 \pm 2.3$ \\
Corn oil (1 ml/kg/i. p.) & $1.3 \pm 0.1$ & $61.1 \pm 3.3$ & $119.8 \pm 3.9$ \\
Fibrosis control CCl with corn oil (1:1) (2 ml/kg/i. p.) & $19.9 \pm 1.6^{\mathrm{a}, \mathrm{b}}$ & $18.9 \pm 1.6^{\mathrm{a}, \mathrm{b}}$ & $47.0 \pm 3.5^{\mathrm{a}, \mathrm{b}}$ \\
Silymarin (100 mg/kg/p. o.) & $13.1 \pm 0.6^{\mathrm{a}, \mathrm{b}, \mathrm{c}}$ & $35.7 \pm 4.0^{\mathrm{a}, \mathrm{b}, \mathrm{c}}$ & $102.8 \pm 3.4^{\mathrm{c}}$ \\
Hesperidin (200 mg/kg/p. o.) & $10.1 \pm 0.7^{\mathrm{a}, \mathrm{b}, \mathrm{c}}$ & $37.5 \pm 2.7^{\mathrm{a}, \mathrm{b}, \mathrm{c}}$ & $104.1 \pm 7.5^{\mathrm{c}}$ \\
\hline
\end{tabular}

Each value represents the mean of 8 animals \pm standard error of the mean (SEM), aSignificantly different from control group value at $p<0.05$, ${ }^{b}$ Significantly different from corn oil group value at $p<0.05$, 'Significantly different from fibrosis control group value at $p<0.05$ and ${ }^{\mathrm{d} S i g n i f i c a n t l y}$ different from silymarin reference treated group value at $p<0.05$

Table 3: Effect of silymarin or hesperidin on serum level of TNF- $\alpha$, liver MPO activity and relative liver weight in rats with experimentallyinduced liver fibrosis

\begin{tabular}{llll}
\hline $\begin{array}{l}\text { Parameters } \Rightarrow \\
\text { Groups } \mathbb{~}\end{array}$ & MPO (u/mg ptn) & TNF $\boldsymbol{\alpha}$ (pg/ml) & Relative liver/Body weight \\
\hline Control (5 ml saline/kg/p. o.) & $2.9 \pm 0.2$ & $31.1 \pm 1.8$ & $3.1 \pm 0.2$ \\
Corn oil (1 ml/kg/i. p.) & $2.7 \pm 0.1$ & $31.1 \pm 1.1$ & $3.5 \pm 0.1$ \\
Fibrosis control CCl with corn oil (1:1) (2 ml/kg/i. p.) & $16.7 \pm 1.4^{\mathrm{a}, \mathrm{b}}$ & $130.4 \pm 5.6^{\mathrm{a}, \mathrm{b}}$ & $4.9 \pm 0.2^{\mathrm{a}, \mathrm{b}}$ \\
Silymarin (100 mg/kg/p. o.) & $8.5 \pm 0.9^{\mathrm{a}, \mathrm{b}, \mathrm{c}}$ & $92.7 \pm 2.5^{\mathrm{a}, \mathrm{b}, \mathrm{c}}$ & $3.7 \pm 0.2^{\mathrm{c}}$ \\
Hesperidin (200 mg/kg/p. o.) & $4.0 \pm 0.7^{\mathrm{c}, \mathrm{d}}$ & $73.4 \pm 2.8^{\mathrm{a}, \mathrm{b}, \mathrm{c}, \mathrm{d}}$ & $3.9 \pm 0.1^{\mathrm{a}, \mathrm{c}}$ \\
\hline
\end{tabular}

Each value represents the mean of 8 animals \pm standard error of the mean (SEM), aSignificantly different from control group value at $p<0.05$, bSignificantly different from corn oil group value at $p<0.05$, 'Significantly different from fibrosis control group value at $p<0.05$ and dSignificantly different from silymarin reference treated group value at $p<0.05$ 
Table 4: Effect of silymarin or hesperidin on serum levels of Cholesterol, TGs, HDL and LDL in rats with experimentally-induced liver fibrosis

\begin{tabular}{|c|c|c|c|c|}
\hline $\begin{array}{l}\text { Parameters } \Rightarrow \\
\text { Groups } \mathbb{}\end{array}$ & Cholesterol (mg/dl) & TGs (mg/dl) & HDL (mg/dl) & LDL (mg/dl) \\
\hline Control (5 ml saline/kg/p. o.) & $145.11 \pm 5.86$ & $89.09 \pm 6.06$ & $61.30 \pm 1.00$ & $65.99 \pm 5.76$ \\
\hline Corn oil ( $1 \mathrm{ml} / \mathrm{kg} /$ i. p.) & $144.01 \pm 6.30$ & $82.80 \pm 3.36$ & $63.08 \pm 1.46$ & $64.37 \pm 6.32$ \\
\hline Fibrosis control $\mathrm{CCl}_{4}$ with corn oil (1:1) $(2 \mathrm{ml} / \mathrm{kg} / \mathrm{i} . \mathrm{p})$. & $236.80 \pm 13.40^{\mathrm{a}, \mathrm{b}}$ & $133.40 \pm 3.37^{a, b}$ & $26.44 \pm 1.68^{\mathrm{a}, \mathrm{b}}$ & $183.68 \pm 14.15^{\mathrm{a}, \mathrm{b}}$ \\
\hline Silymarin (100 mg/kg/p. o.) & $191.31 \pm 3.41^{\mathrm{a}, \mathrm{b}, \mathrm{c}}$ & $99.78 \pm 3.33^{c}$ & $38.80 \pm 1.34^{\mathrm{a}, \mathrm{b}, \mathrm{c}}$ & $122.53 \pm 4.07 \mathrm{a}, \mathrm{b}, \mathrm{c}$ \\
\hline Hesperidin (200 mg/kg/p. o.) & $179.28 \pm 7.09 \mathrm{c}$ & $89.34 \pm 3.16^{c}$ & $41.08 \pm 1.66^{\mathrm{a}, \mathrm{b}, \mathrm{c}}$ & $113.08 \pm 5.58^{\mathrm{a}, \mathrm{b}, \mathrm{c}}$ \\
\hline
\end{tabular}

Each value represents the mean of 8 animals \pm standard error of the mean (SEM), aSignificantly different from control group value at $p<0.05$, bSignificantly different from corn oil group value at $p<0.05$, cSignificantly different from fibrosis control group value at $p<0.05$ and ${ }^{\mathrm{d}}$ Significantly different from silymarin reference treated group value at $p<0.05$

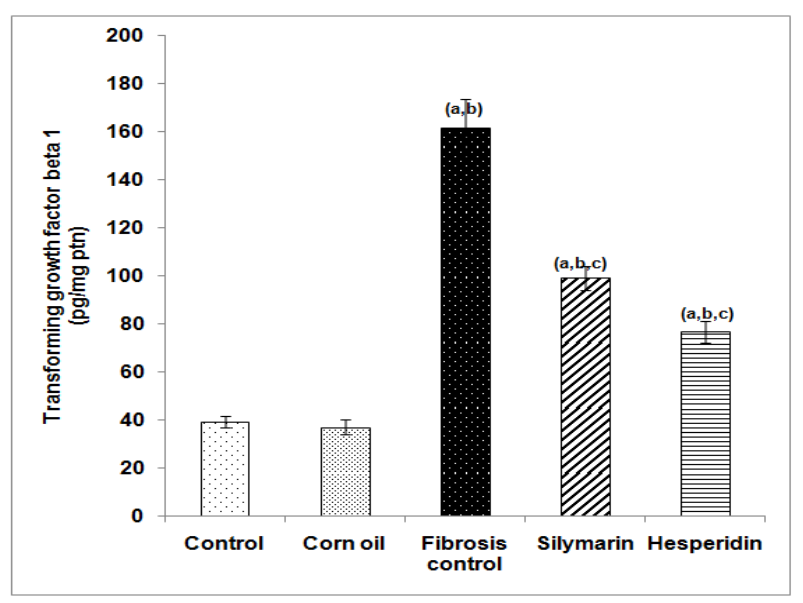

Fig. 1: Effect of silymarin or hesperidin on hepatic content of TGF- $\beta_{1}$ in rats with experimentally induced liver fibrosis

Each value represents the mean of 8 animals \pm standard error of the mean (SEM)

aSignificantly different from control group value at $p<0.05$, bSignificantly different from corn oil group value at $p 0.05$, cSignificantly different from fibrosis control group value at $p<0.05$ and ${ }^{\mathrm{d}}$ Significantly different from silymarin reference treated group value at $p<0.05$

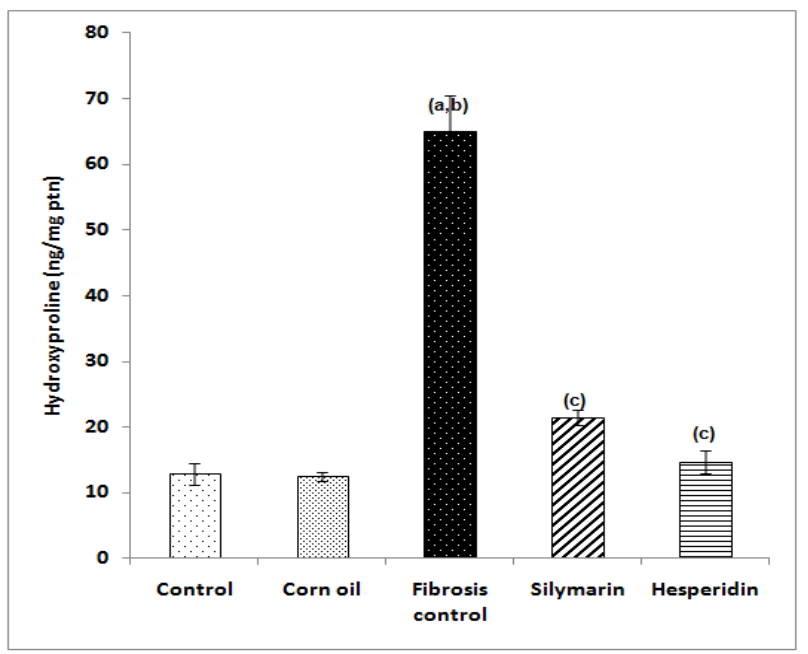

Fig. 2: Effect of silymarin or hesperidin on hepatic content of HYP in rats with experimentally-induced liver fibrosis

Each value represents the mean of 8 animals \pm standard error of the mean (SEM).

aSignificantly different from control group value at $p<0.05$, bSignificantly different from corn oil group value at $p<0.05$, cSignificantly different from fibrosis control group value at $p<0.05$ and ${ }^{d}$ Significantly different from silymarin reference treated group value at $p<0.05$

\section{Histopathological study}

Liver sections obtained from the control and corn oil groups showed normal hepatic architecture with central vein and radiating cords of normal hepatocytes with central rounded vesicular nuclei and prominent nucleoli. Hepatic cords are separated by blood sinusoids lined with endothelium and Von-Kupffer cells (fig. 3a, b). $\mathrm{CCl}_{4}$ group showed increased fibrous tissue with dilated blood vessels. In addition to dilated congested blood sinusoids and activated Von Kupffer cells (fig. 3c, d). However, Silymarin treated group showed normal acidophilic hepatocytes with vesicular nuclei. Somewhat dilated central vein and von kupffer cells can also be noticed (fig. 3e). Hesperidin treated group showed normal central vein with slightly dilated congested blood sinusoids. Most hepatocytes are normal with acidophilic cytoplasm and vesicular nuclei, activation of Von Kupffer cells can be also observed (fig. 3f).

\section{Immunohistochemical study}

Immunostaining for [complete name] ( $\alpha$-SMA) antigen in the liver showing minimal expression of $\alpha$-SMA in the portal area of control and corn oil groups (fig. 4a, b) respectively, whereas HSCs strongly positive of $\alpha$-SMA in the portal areas and along the fibrous septa around the hepatic lobule were observed in group of $\mathrm{CCl}_{4}$-intoxicated group (fig. 4c, d).
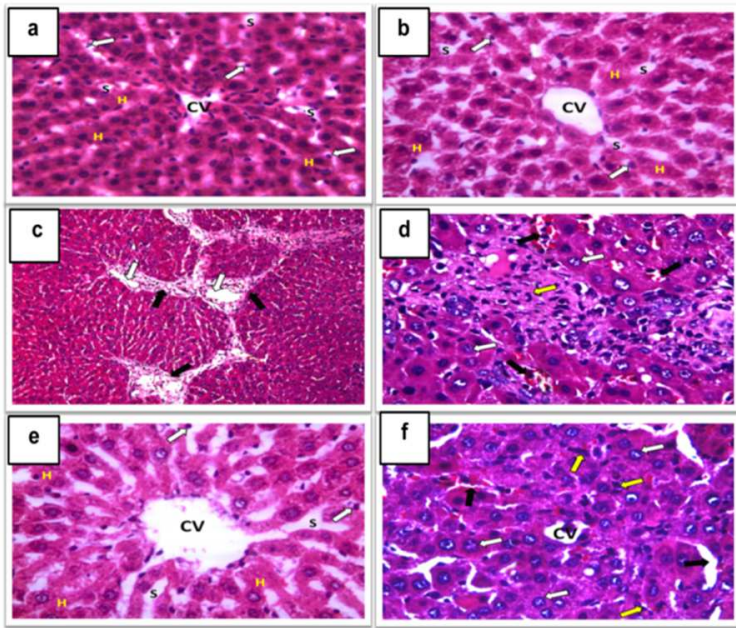

Fig. 3: Histopathological examination of liver sections stained with $H$ and $E(\times 400)$ in normal and treated groups represented as (a) normal control group ( $C V$ : central vein, $H$ : hepatocytes, $S$ : sinusoids, white arrow: von-kupffer cells), (b) corn oil group (CV: central vein, $\mathrm{H}$ : hepatocytes, $S$ : sinusoids, white arrow: vonkupffer cells), (c) fibrosis control group $\mathrm{CCl}_{4}$ in corn oil HandE $(\times 100)$ (black arrow: fibrous tissue, white arrow: dilated blood vessels), (d) fibrosis control group (black arrow: dilated congested blood sinusoids and activated von-kupffer cells, yellow arrow: fibrous tissue), (e) $\mathrm{CCl}_{4}$-intoxicated rat co-treated with silymarin group (CV: central vein, $\mathrm{H}$ : hepatocytes, $\mathrm{S}$ : sinusoids, white arrow: von-kupffer cells) and (f) $\mathrm{CCl}_{4-}$ intoxicated rat co-treated with hesperidin group (CV: central vein, black arrow: blood sinusoids, white arrow: hepatocytes with vesicular nuclei, yellow arrow: von kupffer cells). 
Section of a liver obtained from $\mathrm{CCl}_{4}$-intoxicated group co-treated with silymarin and hesperidin showing moderate positive expression of $\alpha$ SMA in the portal areas and along the incomplete fibrous septa around the hepatic lobules (fig. 4e, f) respectively. Compared with $\mathrm{CCl}_{4}$ intoxicated group, liver of rats treated concomitantly with silymarin and hesperidin showed markedly reduced $\alpha$-SMA positive HSCs.
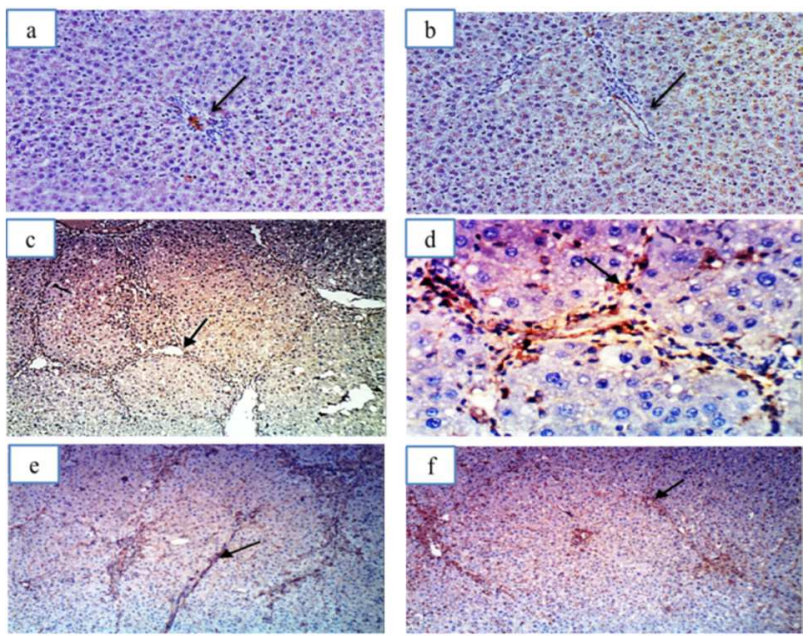

Fig. 4: Examination of alpha-smooth muscle actin ( $\alpha$-SMA) antigen by immunohistochemical staining (magnification $\times 100$ ) in liver sections represented as (a) normal control group (b) corn oil group (c) fibrosis control group $\mathrm{CCl}_{4}$ in corn oil (d) fibrosis control group (magnification $\times 400)(e) \mathrm{CCl}_{4}$-intoxicated rat co-treated with silymarin group and (f) $\mathrm{CCl}_{4}$-intoxicated rat co-treated with hesperidin group

\section{DISCUSSION}

In the present study, hesperidin was evaluated regarding its possible beneficial effect on $\mathrm{CCl}_{4}$-induced liver fibrosis in adult male Wistar albino rats as compared to the reference drug silymarin.

Carbon tetrachloride is an industrial solvent and one of the most commonly experimental models used in the induction of liver fibrosis and for the screening of hepatoprotective agents [28]. It is considered as a toxic chemical that induces hepatotoxicity including fibrosis, fatty degeneration, inflammation, carcinogenicity and hepatocellular death [29].

In the current study, $\mathrm{CCl}_{4}$ intoxication induced a significant increase in serum levels of AST, ALT and total bilirubin which are the most sensitive biomarkers used in the diagnosis of liver injury and hepatic necrosis. These results are in full agreement with Li et al. [30]. During the hepatocellular damage, these enzymes are released into the blood flow from the cytoplasm after the rupture of the hepatic plasma membrane. In addition, $\mathrm{CCl}_{4}$ causes a destruction of hepatic cells and blocking of bile ducts which lead to an increase in serum total bilirubin levels [13]. In addition, $\mathrm{CCl}_{4}$ produced a significant decrease in serum level of albumin, the most important protein synthesised in the liver. This is in accordance with [30] who considered this as an indication of hepatocyte damage and loss of functional integrity.

The protective potential of hesperidin against liver fibrosis was evidenced in this study from its ability to significantly suppress serum levels of liver function markers (ALT, AST and total bilirubin) and to significantly increase serum level of albumin, revealing its hepatoprotective nature against $\mathrm{CCl}_{4}$ hepatotoxicity. This comes in agreement with the results of Elshazly and Mahmoud [9], who reported that hesperidin evoked a hepatoprotective effect against dimethylnitrosamine-induced fibrosis in rats, which is likely attributed to its antioxidant and antiapoptotic effects. These effects would reduce HSCs activation and the progression of fibrosis.

According to the findings of this study, $\mathrm{CCl}_{4}$ intoxication led to a significant decrease in the activity of the antioxidant enzyme catalase, depletion of hepatic GSH content and a significant increase in the hepatic content of MDA. These results are in accordance with those of Tirkey et al. [31] who reported that $\mathrm{CCl}_{4}$ induces a marked oxidative stress in rat liver. Our observations obviously suggested that the oxidative damage might explain at least in part the $\mathrm{CCl}_{4}$ induced liver fibrosis.

The main sources of oxidants in the liver are phagocytes and inflammatory state mediators which are present in the tissues of patients with liver diseases and could generate oxidants upon activation. Oxygen radical production increased lipid peroxidation, a process of oxidative conversion of polyunsaturated fatty acids to products known as MDA or lipid peroxides. Malondialdehyde has high cytotoxicity and inhibitory action on protective enzymes causing it to act as a tumour promoter and co-carcinogenic agent [32].

Regarding the oxidative stress biomarkers, results of the present investigation declared that hesperidin restored the normal values of GSH and MDA contents and CAT activity in the liver confirming its antioxidant potential. These results are in harmony with those of Pari et al. [8] who reported that hesperidin may play a protective role in reducing the toxic effects of iron-induced oxidative damage in liver and kidney, which could be due to its antioxidant potential by scavenging the free radicals.

In this investigation, the ability of hesperidin to improve these oxidative stress biomarkers reflects its antioxidant properties, its ability to suppress lipid peroxidation, and its good free radical scavenging properties.

Data of the current study showed that $\mathrm{CCl}_{4}$ increases the hepatic MPO activity, TNF $\alpha$ and the relative liver weight to all rat body weight. According to the results obtained from our research, we realised that hesperidin was able to significantly suppress the hepatic MPO activity, which is a good indicator for neutrophil infiltration and tissue inflammation and to significantly suppress the serum level of the pro-inflammatory cytokine TNF $\alpha$, which is one of the most important cytokines released during liver fibrosis. In agreement with our results, [33] Fouad et al. inferred the antiinflammatory activity of hesperidin from its ability to reduce the release of inflammatory cytokines like TNF $\alpha$ and cyclooxygenase enzymes, which catalyse a key step in the conversion of arachidonate to prostaglandin (PGs) that plays a critical role in inflammation. Therefore, it could be concluded that inhibition of $\mathrm{COX}_{2}$ will suppress the production of inflammatory prostaglandins.

Myeloperoxidase was found to catalyse the reaction between chloride and hydrogen peroxide to generate hypochlorous acid and other reactive oxidants. The production of these oxidants beside the cytokines will damage the hepatocytes and results in fibrosis [34]. Therefore, the antifibrotic action of hesperidin may in part be explained by its anti-inflammatory potential.

Results of the current study suggested that hesperidin reduced the amount of fibrous tissue in the liver as presented by its ability to reduce the relative liver weight as compared to the fibrosis control group. Therefore, it may be suggested that hesperidin could alleviate hepatic injury caused by $\mathrm{CCl}_{4}$ partly through suppression of the inflammatory response.

It has been previously reported that high cholesterol diet leads to rapid deposition of lipid droplets in the liver. Hypercholesterolemia should be considered as a risk factor for hepatic damage as well as atherosclerosis and coronary artery disease [35].

Results of the current investigation revealed that $\mathrm{CCl}_{4}$ increased serum levels of cholesterol, triglycerides and LDL-Ch, while it produced a significant decrease in serum level of HDL-Ch. Our observations add further evidence for the previous reports of [36] who reported that $\mathrm{CCl}_{4}$ increases lipid profile through cellular oxidative stress, one of the main causes of hepatic lesions produced by $\mathrm{CCl}_{4}$ mediated by the free radicals derived from this toxic substance. The intensification of the oxidative stress enhances the influence of non-essential fatty acids which, in turn, increase the serum and tissue levels of cholesterol and triglycerides. 
Results of the present study showed that hesperidin significantly reduced serum levels of lipid biomarkers such as total cholesterol, triglycerides and LDL cholesterol, while it produced a significant elevation in serum level of HDL cholesterol, probably through the antioxidant mechanism exerted by the flavones in hesperidin. It has been shown that antioxidants and flavonoids can act as inhibitors of lipid peroxidation by neutralising the radicals of polyunsaturated fatty acids and by interrupting the chain reactions, suppress the influence of non-essential fatty acids which, in turn, decrease the serum and tissue levels of cholesterol and triglycerides [36].

Similar results were also shown by Wang et al. [37] who stated that hesperidin effectively alleviated the steatosis of fatty liver, adipose tissue, liver weights and serum total cholesterol concentrations in rats fed with high cholesterol diet and play an important role in reducing the risk of cardiovascular disease.

Hepatic fibrosis is initiated by a damage of hepatocytes that lead to a formation of inflammatory cells and activation of kupffer cells, which subsequently lead to enhanced production of profibrotic cytokines such as TNF $\alpha$ and TGF- $\beta$. Activated TGF- $\beta$ activates expression of many ECM proteins and decreases their degradation by matrix metalloproteinases through increasing the level of tissue inhibitor of metalloproteinases [38]. When the overexpression of TGF- $\beta$ is inhibited, the result is a marked improvement of hepatic fibrosis, and for this reason, several inhibitors of TGF- $\beta$ are investigated as potential drug candidates [39].

Data of the current study declared that $\mathrm{CCl}_{4}$ significantly increased the hepatic content of TGF- $\beta_{1}$ and HYP as compared to the normal control group. Our observations add further evidence for the previous reports of Aldaba-Muruato et al. [39] who mentioned that prolonged $\mathrm{CCl}_{4}$ treatment was associated with progressive fibro genesis, even after stopping administration of $\mathrm{CCl}_{4}$.

Results of the current investigation showed that hesperidin significantly suppressed the hepatic content of TGF- $\beta_{1}$ which is a major indicator for liver fibrosis and HYP which serves as a biochemical indicator of collagen production. This suggests that hesperidin might have a protective potential against liver fibrosis. This comes in agreement also with the results of Pérez-Vargas et al. [40] who reported that hesperidin prevents fibrosis through its ability to modulate profibrotic signals.

Importantly for further molecular and clear interpretation, the expression of $\alpha$-SMA through the immunohistochemical study was examined. It was found that a dramatic increase in the expression of $\alpha$-SMA in the $\mathrm{CCl}_{4}$-intoxicated group. This was considered as an evidence of severe liver fibrosis induced by $\mathrm{CCl}_{4}$. It is a good marker for HSCs activation and establishment of liver fibrosis model.

These findings were in line with the previous reports of Domitrović et al. [41] who stated that liver fibrosis was characterised by activated HSC with accelerated proliferation and enhanced production of ECM components. Hepatic stellate cell activation involves the trans-differentiation from a quiescent state into myofibroblast-like cells with the appearance of $\alpha$-SMA and loss of cellular vitamin A storage.

On the other hand, it was noticed that hesperidin decreases the expression of $\alpha$-SMA as compared to the $\mathrm{CCl}_{4}$-treated group. In the agreement, previous investigations [9] showed similar results, they reported that hesperidin suppressed the progression of HSCs activation, which is manifested as a significant reducer of $\alpha$-SMA expression.

In this study, deposition of collagen in the damaged hepatic areas, associated with increased $\alpha$-SMA, indicates that activated HSCs are responsible for the fibrosis seen in $\mathrm{CCl}_{4}$-intoxicated rat.

The aforementioned biochemical results were supported by histopathological improvement observed in the group treated with hesperidin. Hesperidin modulated the severity of hepatic damage caused by $\mathrm{CCl}_{4}$ leading to a further confirmation of its anti-fibrotic effect.

Silymarin has been recorded as a hepatoprotective agent against different toxicants as alcohol, $\mathrm{CCl}_{4}$ or in the case of long uses of many drugs such as acetaminophen [42]. In this study, silymarin showed generally hepatoprotective effects. It attenuated the effect of $\mathrm{CCl}_{4}$ toxicity in all the measured biochemical parameters, which is also supported by histopathological examination. Interestingly, hesperidin showed generally superior hepatoprotective effects compared to silymarin. These results suggest that hesperidin might be more therapeutically beneficial in ameliorating the progression of hepatic fibrosis than silymarin and sheds light on its potential value for treating various liver injuries.

\section{CONCLUSION}

In conclusion, results of the present study indicate that hesperidin significantly ameliorated $\mathrm{CCl}_{4}$-induced liver fibrosis in rats based on its antioxidant, anti-inflammatory, antilipidemic and anti-fibrotic activities. These effects would reduce HSCs activation and the progression of fibrosis. The effects of hesperidin were not only comparable to but also even better than silymarin, making it a potential therapeutic option from natural sources to prevent liver fibrosis induced by $\mathrm{CCl}_{4}$.

\section{AUTHORS CONTRIBUTION}

ASMAA RAMADAN ABD EL-STTAR: Design of the work, data collection, and writing the manuscript.

MARWA MAHMOUD KHALAF: Data analysis and interpretation, drafting the article.

AMIRA M. ABOYOUSSEF: Critical revision of the article.

ALI AHMED ABOSAIF: Final approval of the version to be published. CONFLICT OF INTERESTS

\section{Declared none}

\section{REFERENCES}

1. Aoudjehane L, Boelle PY, Bisch G, Delelo R, Paye F, Scatton O, et al. Development of an in vitro model to test antifibrotic drugs on primary human liver myofibroblasts. Lab Invest 2016;96:672-9.

2. Achmad A, Fadiah RA, Mustofa, Prihadi AL. The incidence of liver fibrosis based on non-invasive markers and hepatotoxic drug used in hepatitis b patients. Asian J Pharm Clin Res 2014;7:287-90.

3. Mohamed SH, Elbastawisy YM. Efficacy of curcumin in protecting the rat liver from $\mathrm{CCl}_{4}$-induced injury and fibrogenesis. Histological and immunohistochemical study. Life Sci J 2013;10:2824-35.

4. Brenner DA. Molecular pathogenesis of liver fibrosis. Trans Am Clin Climatol Assoc 2009;120:361-8.

5. Essawy AE, Abdel-moneim AM, Khayyat LI, Elzergy AA. Nigella sativa seeds protect against hepatotoxicity and dyslipidemia induced by carbon tetrachloride in mice. J Appl Pharm Sci 2012;2:21-5.

6. Ohta Y, Sahashi D, Sasaki E, Ishiguro I. Alleviation of carbon tetrachloride-induced chronic liver injury and related dysfunction by L-tryptophan in rats. Ann Clin Biochem 1999;36:504-10.

7. El-Demerdash E, Abdel Salam OM, El-Batran SA, Abdallah HMI, Shaffie NM. Inhibition of the renin-angiotensin system attenuates the development of liver fibrosis and oxidative stress in rats. Clin Exp Pharmacol Physiol 2008;35:159-67.

8. Pari L, Karthikeyan A, Karthika P, Rathinam A. Protective effects of hesperidin on oxidative stress, dyslipidaemia and histological changes in iron-induced hepatic and renal toxicity in rats. Toxicol Reports 2014;2:46-55.

9. Elshazly SM, Mahmoud AAA. Antifibrotic activity of hesperidin against dimethylnitrosamine-induced liver fibrosis in rats. Naunyn Schmiedebergs Arch Pharmacol 2014;387:559-67.

10. Barnes S. Nutritional genomics, polyphenols, diets, and their impact on dietetics. J Am Diet Assoc 2008;108:1888-95.

11. Ghosh A, Ghosh T, Jain S. Silymarin-a review on the pharmacodynamics and bioavailability enhancement approaches. J Pharm Sci Technol 2010;2:348-55.

12. Hui AY, Leung WK, Yuen Chan HL, Chan FKL, Yin Go MY, Chan $\mathrm{KK}$, et al. Effect of celecoxib on experimental liver fibrosis in rat. Liver Int 2006;26:125-36. 
13. Shah B, Shah G. Antifibrotic effect of heparin on liver fibrosis model in rats. World J Gastrointest Pharmacol Ther 2012;3:86-92.

14. Reitman S, Frankel S. A colorimetric method for the determination of serum glutamic oxalacetic and glutamic pyruvic transaminases. Am J Clin Pathol 1957;28:56-63.

15. Doumas BT, Watson WA, Biggs HG. Albumin standards and the measurement of serum albumin with Bromocresol Green. Clin Chim Acta 1997;258:21-30.

16. Malloy HT, Evelyn KA. The determination of bilirubin with the photoelectric colorimeter. J Biol Chem 1937;119:481-90.

17. Brouckaert P, Libert C, Everaerdt B, Takahashi N, Cauwels A, Fiers W. Tumor necrosis factor, its receptors and the connection with interleukin 1 and interleukin 6 Immunobiology 1993;187:317-29.

18. Watson D. A simple method for the determination of serum cholesterol. Clin Chim ACTA 1960;5:637-43.

19. Fossati P, Prencipe L. Serum triglycerides determined colorimetrically with an enzyme that produces hydrogen peroxide. Clin Chem 1982;28:2077-80.

20. Castelli WP, Doyle JT, Gordon T, Hames CG, Hjortland MC, Hulley SB, et al. HDL cholesterol and other lipids in coronary heart disease. The cooperative lipoprotein phenotyping study. Circulation 1977;55:767-72.

21. Friedewald WT, Levy RI, Fredrickson DS. Estimation of the concentration of low-density lipoprotein cholesterol in plasma, without the use of the preparative ultracentrifuge. Clin Chem 1972;18:499-502.

22. Blanchette F, Day R, Dong W, Laprise MH, Dubois CM. TGF beta1 regulates gene expression of its own converting enzyme furin. J Clin Invest 1997;99:1974.

23. Patiyal SN, Katoch SS. BETA-adrenoceptor agonist clenbuterol down-regulates matrix metalloproteinase (MMP-9) and results in an impairment of collagen turnover in mice left ventricle. Japan J Physiol 2005;55:165-72.

24. Satoh K. Serum lipid peroxide in cerebrovascular disorders determined by a new colorimetric method. Clin Chim acta 1978;90:37-43.

25. Beutler E, Duron 0, Kelly BM. Improved method for the determination of blood glutathione. J Lab Clin Med 1963;61:882-8.

26. Aebi H. Catalase in vitro. Methods Enzymol 1984;105:121-6.

27. Weiss SJ, Klein R, Slivka A, Wei M. Chlorination of taurine by human neutrophils: evidence for a hypochlorous acid generation. J Clin Invest 1982;70:598.

28. Hassan SK, Mousa AM, El-sammad NM. Attenuation of carbon tetrachloride and ethanol-induced hepatic fibrosis in rats by calligonum comosum shoot extract. Asian J Pharm Clin Res 2017;10:83-91.

29. Hou YL, Tsai YH, Lin YH, Chao JCJ. Ginseng extract and ginsenoside $\mathrm{Rb} 1$ attenuate carbon tetrachloride-induced liver fibrosis in rats. BMC Complementary Altern Med 2014;14:415.
30. Li R, Guo W, Fu Z, Ding G, Zou Y, Wang Z. Hepatoprotective action of Radix Paeoniae Rubra aqueous extract against $\mathrm{CCl}_{4}$ induced hepatic damage. Molecules 2011;16:8684-93.

31. Tirkey N, Pilkhwal S, Kuhad A, Chopra K. Hesperidin, a citrus bioflavonoid, decreases the oxidative stress produced by carbon tetrachloride in rat liver and kidney. BMC Pharmacol 2005;5:1-8.

32. Czeczot H, Scibior D, Skrzycki M, Podsiad M. Glutathione and GSH-dependent enzymes in patients with liver cirrhosis and hepatocellular carcinoma. Acta Biochim Pol 2006;53:237-41.

33. Fouad AA, Albuali WH, Jresat I. Protective effect of hesperidin against cyclophosphamide hepatotoxicity in rats. Int J Biol Biomol Agric Food Biotechnol Eng 2014;8:730-3.

34. Reynolds WF, Patel K, Pianko S, Blatt LM, Nicholas JJ, McHutchison JG. A genotypic association implicates myeloperoxidase in the progression of hepatic fibrosis in chronic hepatitis C virus infection. Genes Immun 2002;3:345-9.

35. Jeong WI, Jeong DH, Do SH, Kim YK, Park HY, Kwon OD, et al. Mild hepatic fibrosis in cholesterol and sodium cholate diet-fed rats. J Vet Med Sci 2005;67:235-42.

36. Andritoiu CV, Ochiuz L, Andritoiu V, Popa M. Effect of apitherapy formulations against carbon tetrachloride-induced toxicity in Wistar rats after three weeks of treatment. Molecules 2014;19:13374-91.

37. Wang X, Hasegawa J, Kitamura Y, Wang Z, Matsuda A, Shinoda $\mathrm{W}$, et al. Effects of hesperidin on the progression of hypercholesterolemia and fatty liver induced by highcholesterol diet in rats. J Pharmacol Sci 2011;117:129-38.

38. Gressner AM, Weiskirchen R, Breitkopf K, Dooley S. Roles of TGF-beta in hepatic fibrosis. Front Biosci 2002;7:793-807.

39. Aldaba-Muruato LR, Moreno MG, Shibayama M, Tsutsumi V, Muriel P. Allopurinol reverses liver damage induced by chronic carbon tetrachloride treatment by decreasing oxidative stress, TGF- $\beta$ production and NF- $\kappa$ B nuclear translocation. Pharmacology 2013;92:138-49.

40. Pérez-Vargas JE, Zarco N, Shibayama M, Segovia J, Tsutsumi V, Muriel P. Hesperidin prevents liver fibrosis in rats by decreasing the expression of nuclear factor- $\kappa \mathrm{B}$, transforming growth factor- $\beta$ and connective tissue growth factor. Pharmacology 2014;94:80-9.

41. Domitrović R, Jakovac H, Tomac J, Šain I. Liver fibrosis in mice induced by carbon tetrachloride and its reversion by luteolin. Toxicol Appl Pharmacol 2009;241:311-21.

42. Kidd PM. Bioavailability and activity of phytosome complexes from botanical polyphenols: the silymarin, curcumin, green tea, and grape seed extracts. Altern Med Rev 2009;14:226-46.

\section{How to cite this article}

- Asmaa Ramadan Abdel-Sttar, Marwa Mahmoud Khalaf, Amira M Aboyoussef, Ali Ahmed Abosaif. Ameliorative effect of hesperidin on carbon tetrachloride-induced liver fibrosis in rats. Int J Pharm Pharm Sci 2017;9(7):45-51 